\title{
Review of: "Immunogenicity and reactogenicity after booster dose with AZD1222 via intradermal route among adult who had received CoronaVac"
}

\author{
Yotam Levin
}

Potential competing interests: The author(s) declared that no potential competing interests exist. The author however discloses his affiliation with NanoPass Technologies Ltd., a developer of intradermal delivery devices.

Thank you for an important manuscript.

The study is of great clinical importance as it further explores strategies for 5 -fold dose-sparing of COVID vaccines in order to dramatically increase available doses for humanity, and especially for low vaccination Low and Middle Income Countries (LMIC).

The study provides a snapshot of fractional dose of a third booster AZ-1222 viral vector vaccine given intradermally, for the eligible Thai population (adults who had received a prime-boost regimen or were inflicted with COVID-19 previously), in order to assess safety, immunogenicity, cellular immunity and, importantly, durability of antibody neutralization.

The results indicate that for the short term (28 days), intradermal delivery of much lesser dose (20\%) generates equivalent immunogenicity (as measured by pseudo neutralization, by the sVNT $80 \%$ method), in a vastly favorable safety profile: while there were more local reactogenicity events reported (primarily self-limiting, transient, erythema and edema), there were far less systemic adverse events: no (0) reports of fever compared with $27 \%$ of reported fever in a parallel IM full dose third booster study, and significantly less complaints of being "feverish", headache, fatigue, myalgia and other AE's. One unsolicited finding unique to intradermal delivery was the occurrence of blisters in $7 \%$ of cases, which warrants additional monitoring in a larger future cohort.

We cannot distinguish the effect of route of delivery vs. reduction in dose on the systemic safety profile since there was no fractional dose IM regimen control, but judging from recent studies in Corona Vaccines intradermally (Intapiboon et al[1]) and other vaccines, the intradermal route is likely to be a major component of the improved systemic profile, especially reduction in myalgia and pain. This may be a very important finding because vaccine adherence, vaccine hesitance as well as the amount of sick days for society, are all affected by (primarily) systemic AE's.

Additionally, cellular T cell immunity markers have shown to be equivalent to the full dose intramuscular control, despite the use of $20 \%$ of the dose. In both routes of delivery, there has been a statistically significant rise in Spike-Nucleoprotein-Membrane-ORF (SNMO)-specific T cell responses, regardless of dose. This is a promising finding, as T cell immunity is considered to be important for reducing severity of disease, and possibly memory. B Cell immunity has followed a similar trend in both fractional dose ID and 
full dose IM delivery; again, showing some promise for potentially functional memory.

Limitations of the study include its size (100 subject per arm); the fact that it is a third boost following two doses of CoronaVac (an inactivated virus vaccine), which has been reported to have lesser vaccine efficacy[2] than some of the mRNA vaccines; Further, the study is limited to adults younger than 60 years old, and data about elderly is of critical public health importance; The results are further limited to nonreplicating viral vector vaccines, following inactivated virus prime boost regimen. Other studies aimed at other vaccines and particularly mRNA vaccines, in the context of a third boost, and across various Variants of Concern (Omicron included) is of critical importance to the research community.

To conclude, it appears that a fractional (20\%) third dose AZ-1222 viral vector vaccine given intradermally induces a non-inferior short-term neutralization, as well as non-inferior T and B cell immunity to that of full dose IM, while having a faster waning of antibody neutralization in 90 days. In the context of vaccine shortage, this takes the work of Cowling[3] et al a notch further: while Cowling advocates that fractional doses (even regardless of route of delivery optimization) are tremendously beneficial to save lives in situations of limited vaccine supply (as very evident in LMIC's), Nantanee et al show that doses could split $5(!)$ times and achieve similar (at least short term) immunity. The regimen should be further improved, for example by using $33 \%$ or $50 \%$ of the IM dose, or by extending the duration between primary regimen and the $3^{\text {rd }}$ boost if this is feasible (which may improve immunogenicity as well[4]). Additional studies are warranted with fractional dose mRNA vaccine intradermally as well. Lastly, we concur with the researchers that intradermal delivery should be conducted with easier to use devices in order to improve reliability and consistency of intradermal vaccination. Such devices exist.

We commend the researchers for their pioneering and important work and wish they continue their exploration of improved immunization regimens for low resource countries which are of great need for dramatically higher vaccine coverage in order to curb the pandemic.

[1] Intapiboon, Porntip, et al. "Immunogenicity and Safety of an Intradermal BNT162b2 mRNA Vaccine Booster after Two Doses of Inactivated SARS-CoV-2 Vaccine in Healthy Population." Vaccines 9.12 (2021): 1375.

[2] Griffin, S. (2021). Covid-19: China's CoronaVac vaccine offers $83.5 \%$ protection against symptomatic infection, interim analysis finds.

[3] Cowling, B.J., Lim, W.W. \& Cobey, S. Fractionation of COVID-19 vaccine doses could extend limited supplies and reduce mortality. Nat Med 27, 1321-1323 (2021). https://doi.org/10.1038/s41591-021-014404

[4] Oxford COVID Vaccine Trial Group. (2021). Single-dose administration and the influence of the timing of the booster dose on immunogenicity and efficacy of ChAdOx1 nCoV-19 (AZD1222) vaccine: a pooled analysis of four randomised trials. The Lancet, 397(10277), 881-891. 
